\title{
Benign pancreatic duct strictures: Medical and endoscopic therapy
}

\author{
JE Geenen MD
}

\begin{abstract}
JE Geenen. Benign pancreatic duct strictures: Medical and endoscopic therapy. Can J Gastroenterol 2000;14(2):127-129. Pancreatic duct strictures usually reflect underlying pancreatic disease and are likely caused by one or more of the following: acute or chronic pancreatitis, benign or malignant pancreatic neoplasm, pseudocyst and trauma.

The characteristics of pancreatic strictures are identified, and medical and endoscopic therapy options are reviewed.
\end{abstract}

Key Words: Endoscopy; Pancreatic cancer; Pancreatic duct strictures; Pancreatitis

\section{Strictures bénignes du canal pancréatique : Traitement médical et endoscopique}

RÉSUMÉ : Les strictures bénignes du canal pancréatique sont en général le reflet d'une maladie pancréatique sous-jacente et on les attribue le plus probablement à l'un ou l'autre des problèmes suivants : pancréatite aiguë ou chronique, néoplasie pancréatique bénigne ou maligne, pseudokyste ou traumatisme. On décrit ici les caractéristiques des strictures pancréatiques et on passe en revue les options thérapeutiques médicales et endoscopiques.
$\mathrm{P}$ ancreatic duct strictures are discovered during endoscopic retrograde cholangiopancreatography and usually reflect underlying pancreatic disease. The finding of a pancreatic duct stricture often poses a dilemma regarding appropriate management. The cause of a pancreatic duct stricture is likely to include one or more of the following: acute or chronic pancreatitis, pancreatic neoplasm (benign or malignant), pseudocyst and trauma. Filling defects such as protein plugs or stones may resemble a stricture. Cancer is the most worrisome cause of a pancreatic duct stricture and should be considered in all patients in whom a pancreatic duct stricture is identified.

When evaluating patients with pancreatic duct strictures of unknown cause, background clinical information is very important. Interpretation of the pancreatic duct stricture requires the physician to integrate all the pertinent information available, including clinical findings, radiological appearance, tissue examination and serum markers for pancreatic cancer.

\section{CHARACTERISTICS OF PANCREATIC DUCT STRICTURES}

Several important features of the pancreatogram can assist the physician when examining the $\mathrm{x}$-ray. Anatomical variations of the pancreatic duct must be considered when evaluating pancreatic duct strictures. These variations include a dominant Santorini, loops or kinks of the duct, and pseudostrictures (which is a normal narrowing at the junction point of the dorsal and ventral duct system). If the endoscopist is aware of these variants, further unnecessary evaluation can be avoided. Changes that should be looked for when examining the pancreatogram include irregularity or dilation of the pancreatic duct or the secondary branches. The appearance of a single stricture with upstream dilation and a normal ductogram downstream to the stricture in a patient without any symptoms or predisposing factors for pancreatitis is most likely due to a tumour. Changes throughout the duct, especially when they occur downstream to the pancreatic stricture in addition to anticipated changes upstream,

This mini-review was prepared from a presentation made at the World Congress of Gastroenterology, September 6 to 11, 1998, Vienna, Austria Medical College of Wisconsin, Pancreatic Biliary Center, St Luke's Medical Center, Milwaukee, Wisconsin, USA

Correspondence: Dr Joseph E Geenen, Gastroenterology Consultants, Ltd, 2801 W Kinnickinnic River Parkway, Suite 570, Milwaukee,

Wisconsin 53215, USA. Telephone 414-649-5200, fax 414-649-5004

Received for publication November 12, 1998. Accepted November 19, 1998 
are suggestive of chronic pancreatitis. Multiple strictures in a 'chain of lakes' appearance are characteristic of chronic pancreatitis. Unfortunately, none of these features supporting a diagnosis of chronic pancreatitis is useful in ruling out pancreatic cancer in the individual patient because pancreatic cancer may develop in a patient with underlying chronic pancreatitis. In fact, there is an increased risk of pancreatic cancer in patients with chronic pancreatitis. Thus, the pancreatogram alone is not enough to distinguish chronic pancreatitis patients with superimposed pancreatic cancer from those without. Aggressive attempts to obtain tissue should be made to establish a diagnosis. A double duct sign refers to the presence of a stricture involving both the bile duct and the pancreatic duct. In the past, the double duct sign was believed to have been pathognomonic for pancreatic cancer. This is no longer believed to be correct. The clinical history, as well as tissue sampling, helps to distinguish chronic pancreatitis from cancer in patients with double duct signs. If the pancreatic duct is completely cut off, there is an abrupt stop in the flow of contrast at some point along the length of the pancreatic duct. Incomplete filling of the pancreatic duct may also produce the appearance of a tapered stricture. Sufficient contrast must be injected so that the secondary branches of the pancreatic duct can be seen downstream to the blockage. This confirms the presence of a functionally important stricture in the main pancreatic duct because it indicates that the contrast has taken the path of least resistance into the secondary branches. Pancreas divisum is an anatomical variation that may mimic a complete cutoff of the pancreatic duct; however, it has a fairly consistent appearance because the duct tapers and secondary branches are usually visualized. True abrupt cutoff of the pancreatic duct almost always suggests underlying pancreatic cancer or pancreatitis associated with a pseudocyst, stricture or pancreatic stone material.

Filling defects such as pancreatic stones and protein plugs may resemble a pancreatic duct stricture. Most of these filling defects can be differentiated with careful attention to technique and the characteristics of the pancreatogram.

Thus, it is important to be aware of the history and clinical presentation, and results of abdominal imaging studies, which can help distinguish strictures secondary to pancreatitis from a pancreatic neoplasm. There is some diagnostic and therapeutic benefit in trying to cross the obstruction using a glide or guidewire, especially in patients with a pseudocyst that may be drained via the transpapillary route. Also, it is helpful to get a guidewire across a stricture to retrieve better cellular material for cytology examination.

\section{DIAGNOSIS}

Although some of the features of the pancreatogram are highly suggestive of malignancy or chronic pancreatitis, it is evident that there are no pathognomonic features to distinguish the two processes in a patient with a pancreatic duct stricture. Therefore, a tissue sample must be taken to make a definitive diagnosis. Thus, in all patients with pancreatic duct strictures, tissue should be obtained, if possible, from the pancreatic duct stricture using brush cytology, a forceps biopsy and endoscopic needle aspiration using the Howell needle. If malignancy is suspected on the basis of the pancreatogram as well as the computed tomography (CT) scan or ultrasound, a CT-guided fine needle aspiration is also recommended. In most patients with chronic pancreatitis and pancreatic duct stricture, CT-guided tissue sampling should also be done. In cases in which there is a concern about underlying pancreatic carcinoma and no clear cut mass on CT scan, the CA19-9 level may not be sufficiently elevated. Thus, a normal or minimally elevated CA19-9 does not exclude pancreatic malignancy, while an excessively high level may suggest a neoplasm.

In summary, the diagnostic strategy includes clinical findings, traditional imaging techniques, tissue sampling and serum markers to help distinguish benign from malignant disease in patients with pancreatic duct strictures.

\section{MEDICAL TREATMENT}

Intractable abdominal pain is the most important indication for the treatment of patients with pancreatic duct strictures and chronic pancreatitis. Knowledge of the pathogenesis of the pain, however, is still fragmentary. The pain is thought to be due to acute inflammatory processes in patients with chronic pancreatitis, increased intrapancreatic duct pressure and perineural inflammation. The treatment objectives in patients with pancreatic duct strictures are to prevent recurrent attacks of acute pancreatitis and alleviate the intractable abdominal pain. Medical therapy to alleviate pancreatic pain includes analgesics, pancreatic enzymes, abstinence from alcohol, a low fat diet with frequent meals and, in some instances, a celiac plexus block. Less than $50 \%$ of patients respond to medical therapy and are referred for endoscopic or surgical therapy.

\section{ENDOSCOPIC TREATMENT}

Endoscopic techniques that provide alternatives for treating pain in chronic pancreatitis have been developed. Among these are insertion of pancreatic stents in either the major or minor papilla to overcome intraductal obstruction caused by duct strictures, pancreatic stones or hypertensive sphincter of Oddi; mechanical retrieval of intraductal stones; and drainage of pancreatic pseudocysts that communicate with the main pancreatic duct. By removing these barriers to the outflow of pancreatic juice, chronic pain and/or exacerbation of chronic pancreatitis may be remedied. Although pancreatic ductal strictures can be treated by catheter or balloon dilation alone, subsequent pancreatic stent insertion is required in most cases because stricture relapse is common. Over 200 patients with pancreatic duct strictures have been treated with dilation and/or stents. The technical success of stricture manipulation was reported to be $70 \%$ to $100 \%$ of these patients with or without prior pancreatic sphincterotomy. Of the reports that provide adequate detail of treatment responses and procedure complications, post-treatment pain alleviation was achieved in the majority of patients during a follow-up of one to 28 months (Table 1). 
TABLE 1

Stent therapy for dominant strictures in patients with chronic pancreatitis

\begin{tabular}{|c|c|c|c|c|c|c|}
\hline \multirow[b]{2}{*}{ Author, year (reference) } & \multicolumn{4}{|c|}{ Improvement } & \multicolumn{2}{|c|}{ Complications } \\
\hline & Number of patients & $\mathbf{n}$ & $\%$ & Follow-up (months) & $\mathbf{n}$ & $\%$ \\
\hline Siegel, 1989 (2) & 44 & 41 & 93 & $1-72$ & - & - \\
\hline Burdick, 1993 (4) & 56 & 38 & 68 & $3-6$ & - & - \\
\hline Ponchon, 1995 (1) & 23 & 12 & 52 & 12 & 9 & 39 \\
\hline Smits, 1995 (5) & 49 & 40 & 82 & $6-128$ & 9 & 18 \\
\hline
\end{tabular}

Although relatively uncommon in most patients, sustained pain relief following treatment has been observed. Interestingly, shorter convincing responses to treatment may predict future benefit from surgical interventions. Finally, the presence of both obstruction and dilation is apparently crucial in predicting which patients benefit from stricture therapy (Table 2). Of the 247 patients with pancreatic duct strictures treated by dilation and/or stent placement, 22 (9\%) developed procedure-related complications. Pancreatic stent occlusion was particularly observed at four to six months, whereas proximal stent migration occurred rarely. Frequent stent occlusion prompted one group of investigators to place self-expanding metal stents across the pancreatic duct stricture. (Some patients were considered to be poor surgical risks for surgical therapy.) The preliminary results of metal stent therapy were not encouraging at the six months' follow-up.

\section{SURGICAL THERAPY}

Surgical intervention has been the classic approach for patients with pain and chronic pancreatitis. Surgical drainage procedures remain technically difficult and even in the best hands have a perioperative morbidity rate of $20 \%$ to $40 \%$ and a mortality rate of $2 \%$ to $5 \%$. Also, pancreaticointestinal anastomoses tend to obstruct over time and, consequently, the long term success rate of pain relief falls to approximately $60 \%$ to $80 \%$.

The results in the literature suggest that a select subgroup of patients with painful chronic pancreatitis may benefit from endoscopic therapy: patients with dominant proximal strictures with dilation of the pancreatic duct; patients with recurrent episodes of pain, not chronic pain; and patients who present with nonalcoholic painful pancreatitis.

\section{REFERENCES}

1. Ponchon T, Bory RM, Hedelius F, et al. Endoscopic stenting for pain relief in chronic pancreatitis: results of a standardized protocol. Gastrointest Endosc 1995;42:452-6.

2. Siegel JH, Pullano WJ, Safrany L. Endoscopic sphincterotomy for acquired pancreatitis: effective long-term management. Gastrointest Endosc 1989;35:168-9.

3. Cremer M, Deviere J, Delhaye M, Baize M, Vandermeeren A. Stenting in severe chronic pancreatitis: results of medium-term follow-up in seventy-six patients. Endoscopy 1991;23:171-6.
TABLE 2

Stent therapy for patients with chronic pancreatitis

\begin{tabular}{lcccc}
\hline Duct morphology & $\begin{array}{c}\text { Number of } \\
\text { patients }\end{array}$ & $\mathbf{n}$ & $\%$ & $\begin{array}{c}\text { Follow-up } \\
\text { (months) }\end{array}$ \\
\hline $\begin{array}{l}\text { No dilation or } \\
\text { obstruction }\end{array}$ & 17 & 8 & 47 & 6 \\
$\begin{array}{l}\text { Obstruction } \\
\text { Dilation }\end{array}$ & 11 & 7 & 64 & 6 \\
$\begin{array}{l}\text { Obstruction and } \\
\quad \text { dilation* }\end{array}$ & 15 & 10 & 67 & 6 \\
\hline
\end{tabular}

*P $=0.003$

Ponchon et al (1) suggested that there are three types of patients who present with painful chronic pancreatitis: patients who probably would not benefit from surgical drainage - those whose pain persists despite adequate reduction in ductal pressure, suggesting other factors contributing to the pain, and drainage procedures, either endoscopic or surgical; patients who are symptom free after final stent removal in whom strictures have disappeared, suggesting a favourable long term outcome; and patients who would benefit from surgical drainage - those who have symptom relief but recurrence of symptoms or ductal strictures.

\section{CONCLUSIONS}

Malignancy has to be considered in all patients. Medical therapy is helpful in a minority of patients. Endoscopic treatment for pain seems to be beneficial in a select group of patients with dominant strictures with proximal dilation, recurrent attacks of pain and nonalcohol etiology.

4. Burdick JS, Geenen JE, Hogan WM, et al. Pancreatic stent therapy in chronic pancreatitis: which patients benefit? Gastrointest Endosc 1993;39:A245. (Abst)

5. Smits ME, Badiga SM, Rauws EAJ, Tytgat GNJ, Huibregtse K. Long-term results of pancreatic stents in chronic pancreatitis. Gastrointest Endosc 1995;42:461-7.

6. Kozarek RA, Ball TJ, Patterson DJ. Endoscopic approach to pancreatic duct calculi and obstructive pancreatitis. Am J Gastroenterol 1992;87:600-3. 


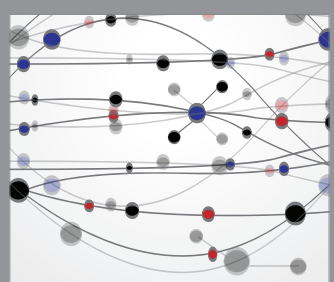

The Scientific World Journal
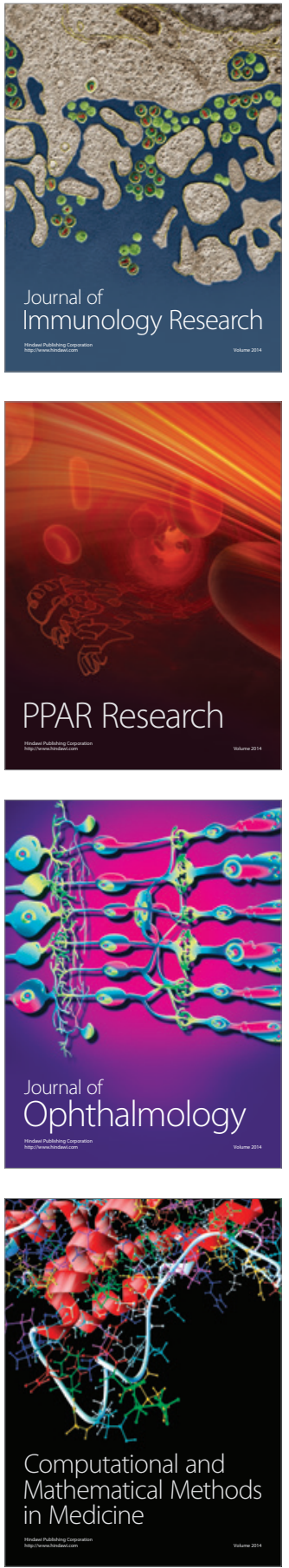

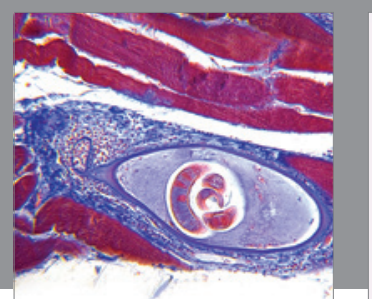

Gastroenterology Research and Practice

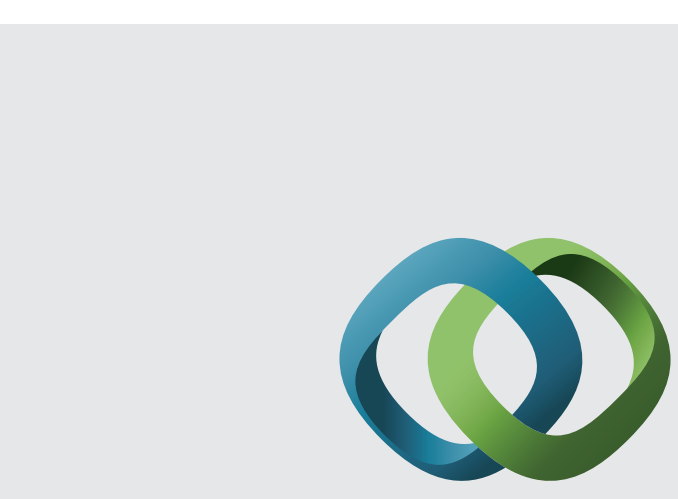

\section{Hindawi}

Submit your manuscripts at

http://www.hindawi.com
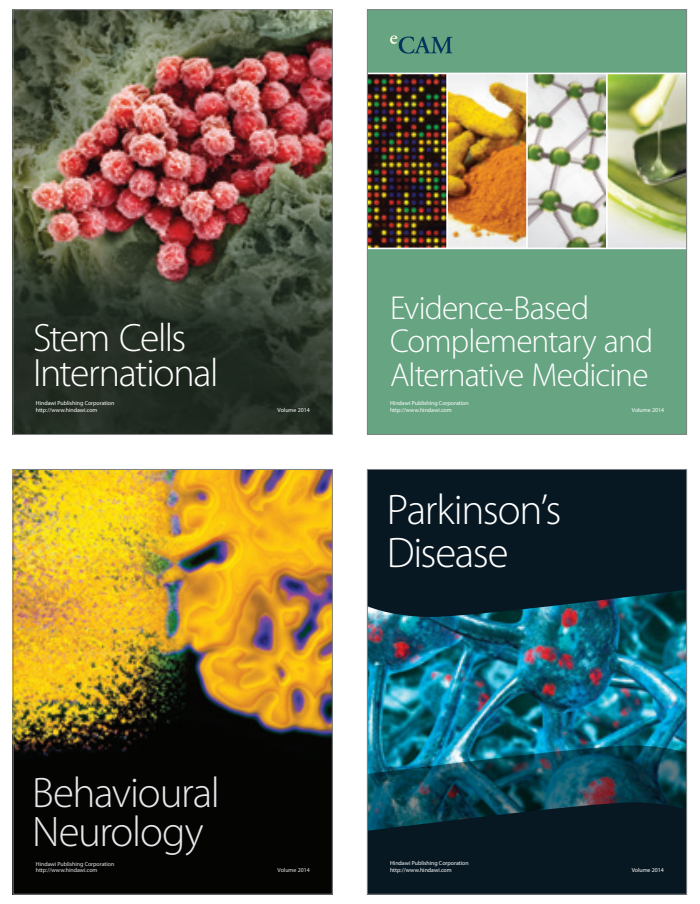
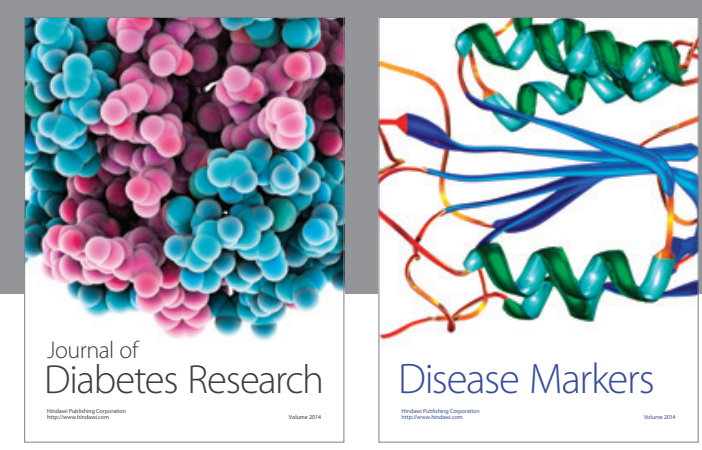

Disease Markers
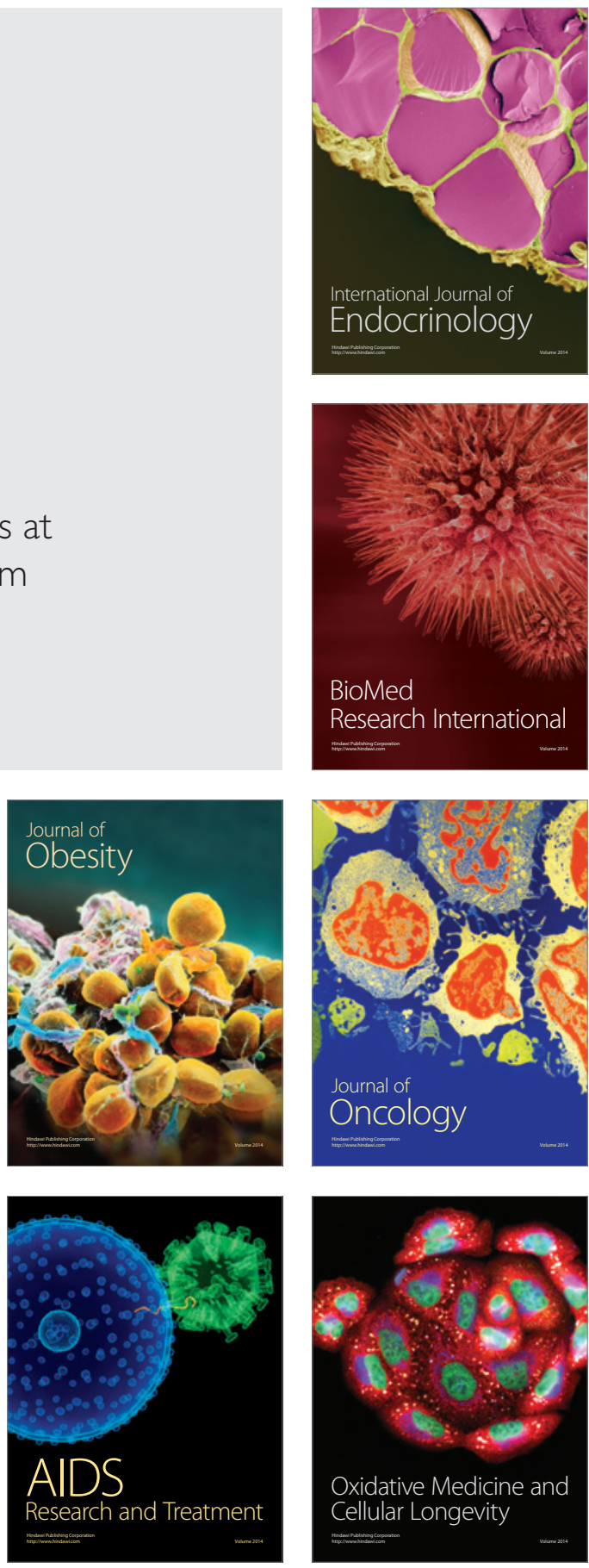